\title{
Chemical Potentials Driving Diffusional Flow in Multilayers
}

\section{Citation}

Spaepen, Frans. 1994. "Chemical Potentials Driving Diffusional Flow in Multilayers." Materials Science Forum 154: 43-54. doi:10.4028/www.scientific.net/msf.154.43.

\section{Published Version}

doi:10.4028/www.scientific.net/msf.154.43

\section{Permanent link}

http://nrs.harvard.edu/urn-3:HUL.InstRepos:26978287

\section{Terms of Use}

This article was downloaded from Harvard University's DASH repository, and is made available under the terms and conditions applicable to Other Posted Material, as set forth at http:// nrs.harvard.edu/urn-3:HUL.InstRepos:dash.current.terms-of-use\#LAA

\section{Share Your Story}

The Harvard community has made this article openly available.

Please share how this access benefits you. Submit a story.

\section{Accessibility}


This is a preprint of a paper published in

Materials Science Forum Vol. 154 (1994) pp. 43-54, Trans Tech Publications, Switzerland.

\title{
Chemical Potentials Driving Diffusional Flow in Multilayers \\ Frans Spaepen \\ Division of Applied Sciences \\ Harvard University, Cambridge, MA 02138, U.S.A.
}

\begin{abstract}
The capillary part of the chemical potential is calculated for three cases: a single anisotropic grain, a thin film with columnar grains, and a polycrystalline textured binary multilayer. A proposal is made for partitioning the contribution arising from the interfacial free energy between the two species.
\end{abstract}

\section{INTRODUCTION}

The classic method for absolute measurement of the surface tension (surface free energy) of a crystal is a zero creep experiment on a wire with a bamboo-type grain structure [1-3], or a thin film with a columnar grain structure [4,5]. The surface tension is determined from the tensile load at which the strain rate is zero. Recently, this method has been applied to textured, polycrystalline multilayers to determine the interfacial tension between the two phases of the repeated bilayer [6-8]. The extraction of a value for the interfacial tension from the zero creep load in this case requires a full analysis of the diffusional creep in the multilayer. The driving forces of the fluxes governing the creep are differences in chemical potential between the faces of the grains, since the grain boundaries and interfaces are the sources and sinks of the diffusing atoms.

The mechanical part of these chemical potentials is well known to be $\sigma \Omega$, where $\sigma$ is the traction normal to the boundary or interface and $\Omega$ is the atomic volume $[9,10]$. The capillary part, which depends on the shape of the grains and the anisotropic interfacial tensions, is not as obvious. The standard method for determining the chemical potential is to find the work, per atom, required to add material to the system at places where it can be incorporated without changing the state of the system: at free surfaces, incoherent boundaries or the cores of dislocations that can climb [11,12].

This paper describes three cases. The equilibrium of an anisotropic crystal is a well known problem that can be related straightforwardly to the chemical potentials derived by adding material to each of the outside faces. The case of a polycrystalline thin film with a columnar grain structure is similar, but the definition of the chemical potential requires the introduction of material as an entire slab along the grain boundaries, throughout the film. This leads to an intuitively obvious "partitioning" of the grain boundary free energy between adjacent grains for the definition of the chemical potential. Finally, the case of a two-component multilayer, with only internal interfaces, is considered. Here it is necessary to partition the capillary contribution from the interphase boundary between the two components, which is not obvious. This is done by determining exactly the equilibrium partitioning, and extending it to non-equilibrium shapes. 


\section{A SINGLE ANISOTROPIC GRAIN}

a) Equilibrium shape

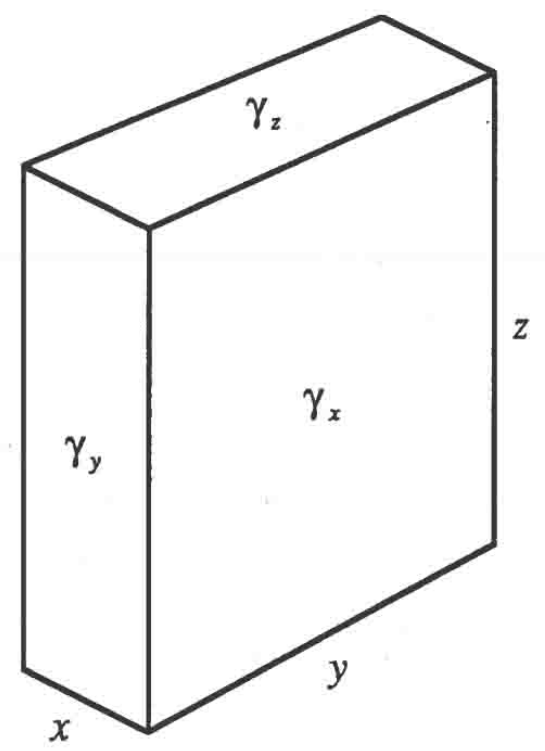

Figure 1: A single anisotropic grain bounded by orthogonal faces with different surface free energies.

The grain of Figure 1 is bounded by orthogonal faces with surface tensions $\gamma_{x}, \gamma_{y}$ and $\gamma_{z}$, and edge lengths $x, y$ and $z$. For simplicity just the monatomic case is considered. The equilibrium shape of the grain is formed by minimizing the total surface free energy

$$
G_{s}=2\left(\gamma_{x} y z+\gamma_{y} x z+\gamma_{z} x y\right)
$$

under the constraint of constant volume

$$
V=x y z
$$

This is most easily done by introducing a Lagrange multiplier $\mu$, to form the function

$$
F=G_{s}+\mu V
$$

This leads to the conditions

$$
\begin{aligned}
& \frac{\partial F}{\partial x}=2\left(\gamma_{y} z+\gamma_{z} y\right)+\mu y z=0 \\
& \frac{\partial F}{\partial y}=2\left(\gamma_{x} z+\gamma_{z} x\right)+\mu x z=0 \\
& \frac{\partial F}{\partial z}=2\left(\gamma_{y} x+\gamma_{x} y\right)+\mu x y=0
\end{aligned}
$$

The equilibrium values $x_{o}, y_{o}$ and $z_{o}$ of the edge lengths then must satisfy the conditions:

$$
\mu=2\left(\frac{\gamma_{y}}{y_{o}}+\frac{\gamma_{z}}{z_{o}}\right)=2\left(\frac{\gamma_{x}}{x_{o}}+\frac{\gamma_{z}}{z_{o}}\right)=2\left(\frac{\gamma_{x}}{x_{o}}+\frac{\gamma_{y}}{y_{o}}\right)
$$


or

$$
\frac{\gamma_{x}}{x_{o}}=\frac{\gamma_{y}}{y_{o}}=\frac{\gamma_{z}}{z_{o}}
$$

which is the well-known result from the Wulff construction $[13,14]$.

b) Chemical potentials

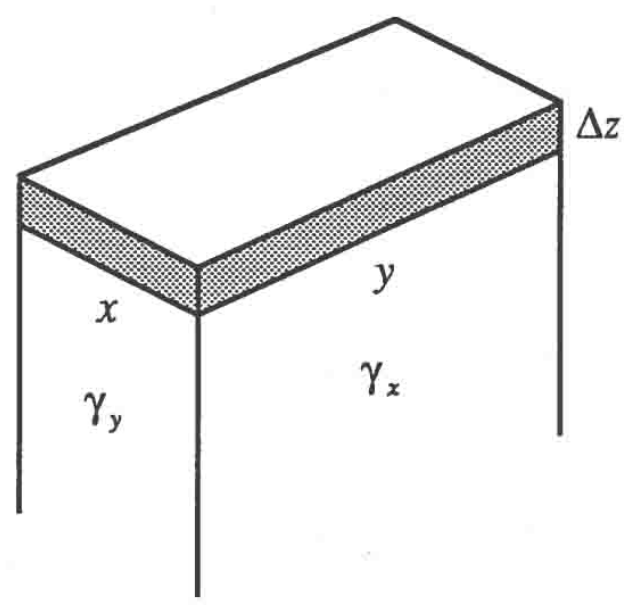

Figure 2: Illustration of the method for determining the chemical potential of the atoms on the z-face. Capillary work is required to form the shaded surfaces.

For an arbitrary shape of the grain of Figure 1, the chemical potential of the atoms on different faces is not the same. Consider adding a layer of thickness $\Delta z$ to the top surface (Figure 2). Its volume is $x y \Delta z$, and the number of atoms in it is then $\Delta n_{z}=x y \Delta z / \Omega$, where $\Omega$ is the atomic volume. The surface work required to form the layer is:

$$
W_{c}=2\left(x \gamma_{y}+y \gamma_{x}\right) \Delta z
$$

The capillary contribution to the chemical potential is, by definition, the surface work per added atom:

$$
\mu_{z}=\frac{W_{c}}{\Delta n_{z}}=2\left(\frac{\gamma_{x}}{x}+\frac{\gamma_{y}}{y}\right) \Omega
$$

Similar expressions are obtained for $\mu_{x}$ and $\mu_{y}$ by cyclic permutation of the subscripts. Note that in equilibrium the three chemical potentials are all equal and that their equilibrium value (per unit volume) is the Lagrange multiplier of Equation (5). If the values of $x, y$ and $z$ do not satisfy the equilibrium condition, the chemical potentials differ, and induce diffusional flow towards the equilibrium shape. For example, if $x=y=z$ and $\gamma_{z}<\gamma_{x}=\gamma_{y}$, Equation (8) and its equivalents give $\mu_{z}>\mu_{x}=\mu_{y}$. Matter then flows from the top surface to the sides. thereby exposing relatively more of the top surface with its low surface tension.

\section{A POLYCRYSTALLINE THIN FILM}

a) Equilibrium conditions 


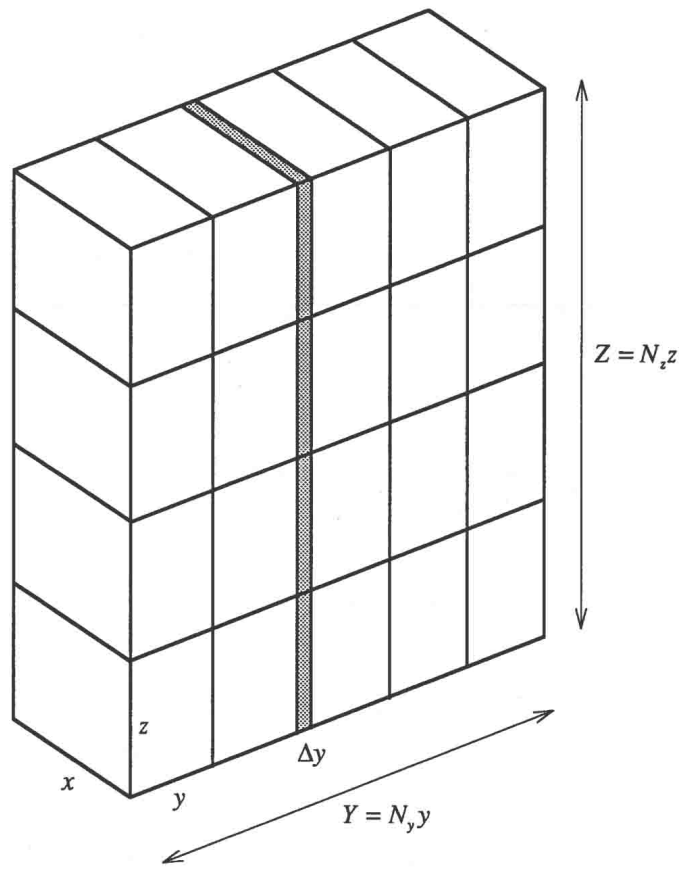

Figure 3: Part of a thin film consisting of identical rectangular grains ( $N_{z}$ and $N_{y}$ are large). The shaded slab illustrates the method for determining the chemical potential of the atoms near the grain boundaries

As illustrated on Figure 3, the film under consideration has a thickness $x$, and contains a large number of identical, rectangular grains of dimensions $y$ and $z$. The interfacial tensions $\gamma_{y}$ and $\gamma_{z}$ are now grain boundary tensions, and $\gamma_{x}$ is still a surface tension. The lateral dimensions of the film are $Y$ and $Z$ with $N_{y}=Y / y$ and $N_{z}=Z / z$. Since $N_{y}$ and $N_{z}$ are large, the number of grains and the number of grain boundaries is assumed equal $\left(N_{y} \approx N_{y}-1\right)$. For the same reason, the contributions of the film edges to the surface free energy can be neglected. The total surface and interface free energy of the system is then:

$$
G_{s}=2 N_{y} N_{z} y z \gamma_{x}+N_{y} Z x \gamma_{y}+N_{z} Y x \gamma_{z}=N_{y} N_{z}\left(2 y z_{x}+x z \gamma_{y}+x y \gamma_{z}\right)
$$

Minimization, again using a Lagrange multiplier, under the conditions that the grain configuration, $N_{y}$ and $N_{z}$, and each grain volume $x y z$ remain constant gives conditions analogous to those of Equations (5) and (6):

or

$$
\mu=\frac{\gamma_{y}}{y_{o}}+\frac{\gamma_{z}}{z_{o}}=\frac{2 \gamma_{x}}{x_{o}}+\frac{\gamma_{z}}{z_{o}}=\frac{2 \gamma_{x}}{x_{o}}+\frac{\gamma_{y}}{y_{o}}
$$

$$
\frac{2 \gamma_{x}}{x_{o}}=\frac{\gamma_{y}}{y_{o}}=\frac{\gamma_{z}}{z_{o}}
$$

\section{b) Chemical potentials}

Two cases need be considered: that of atoms at the free surface and that of atoms near the grain boundaries. For the first case, consider a slab of thickness $\Delta x$ added to one of the free surfaces. Its 
volume is $Y Z \Delta z$ and the number of atoms in it is $\Delta n_{x}=Y Z \Delta x / \Omega$. The capillary work required arises from the extension of the grain boundaries through the slab:

$$
W_{c, x}=N_{z} Y \Delta x \gamma_{z}+N_{y} Z \Delta x \gamma_{y}
$$

which leads to the chemical potential for the free surfaces

$$
\mu_{x}=\frac{W_{c, x}}{\Delta n_{x}}=\frac{\gamma_{y}}{y}+\frac{y_{z}}{z}
$$

For the second case, consider a slab of thickness $\Delta y$ inserted into the film along the grain boundaries as shown in Figure 3. Its volume is $Z x \Delta y$ and the number of atoms in it is $\Delta n_{y}=Z x \Delta y / \Omega$. The capillary work required to insert the slab is

$$
W_{c, y}=N_{z} x \Delta y \gamma_{z}+2 Z \Delta y \gamma_{x}
$$

The first term arises from the extension of one set of grain boundaries, the second term from the creation of new free surface on the front and back. The resulting chemical potential is then:

$$
\mu_{y}=\frac{W_{c, y}}{\Delta n_{y}}=\left(\frac{2 \gamma_{x}}{x}+\frac{\gamma_{z}}{z}\right) \Omega
$$

By symmetry, the expression for $\mu_{z}$ is the same as that for $\mu_{y}$ if $z$ is substituted for $y$. Equations (10), (11), (13) and (15) have the same form as (5), (6) and (8) - their counterparts for the single grain - except that the grain boundary free energies $\gamma_{y}$ and $\gamma_{z}$ are counted for only half the value of their free surface counterparts. For this reason one can simply treat each grain individually, if the grain boundary free energies are partitioned equally between neighboring grains.

\section{A TEXTURED POLYCRYSTALLINE MULTILAYER}

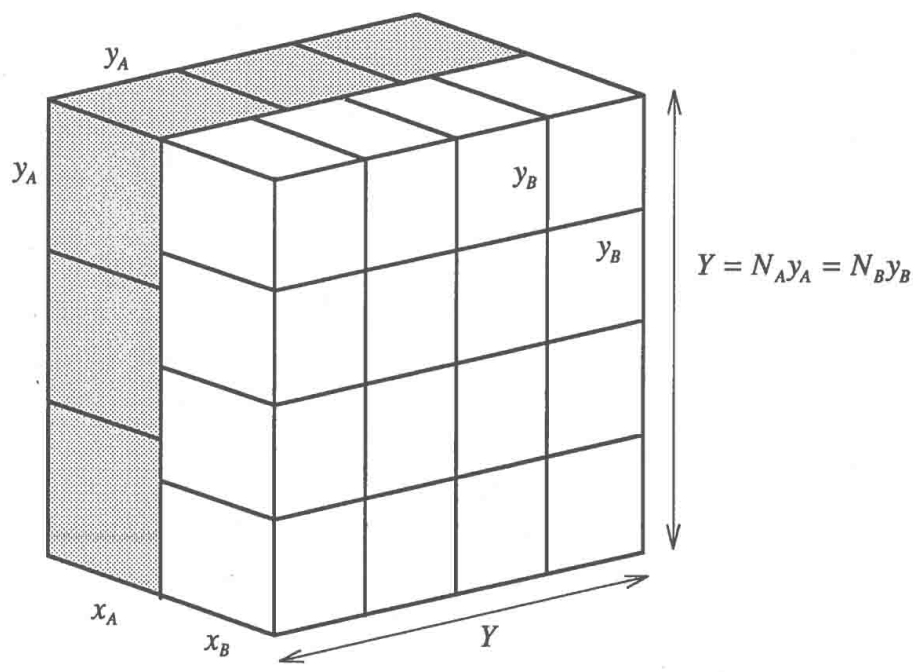

Figure 4: Repeat unit, translated a large number of times in all three directions to form a textured polycrystalline multilayer with square grains of pure $A$ (shaded) and pure $B . N_{A}$ and $N_{B}$ are integers, and can be as small as one.

The multilayer consists of a large number of alternating layers of pure $A$ and pure $B$ (immiscible) of thickness $x_{A}$ and $x_{B}$. As a model of a textured film with equiaxed columnar grains in each 
layer, a single grain boundary free energy is used for each layer: $\gamma_{A}$ and $\gamma_{B}$, and the grains have a square shape of size $y_{A}$ and $y_{B}$ (see Figure 4). The interface between $A$ and $B$ has an interfacial free energy $\gamma_{A B}$. Let $Y$ be the least common multiple of the two grain sizes:

$$
Y=N_{A} y_{A}=N_{B} y_{B}
$$

The structure of Figure 4 is therefore repeated periodically in all three directions. Since the number of interfaces in all orientations is large, the free surfaces can be entirely neglected.

\section{a) Equilibrium conditions}

The total grain boundary and interface free energy of the repeat unit of Figure 4 is:

$$
G_{s}=2 N_{A} Y x_{A} \gamma_{A}+2 N_{B} Y x_{B} \gamma_{B}+2 Y^{2} \gamma_{A B}
$$

Note that the free energies of the outer surfaces of the repeat unit are counted for half their value, as shown in the previous section. This is minimized under the constraints that all grains keep the

same volume $\left(V_{A}=x_{A} y_{A}^{2}\right.$ and $V_{B}=x_{B} y_{B}^{2}$, both constant) and the dimensional relations derived from Equation (16): $C_{1}=Y-N_{A} y_{A}=0$ and $C_{2}=Y-N_{B} Y_{B}=0$. Introducing four Lagrangian multipliers gives the function

$$
F=G_{s}+\lambda V_{A}+\mu V_{B}+\nu C_{1}+\eta C_{2}
$$

Minimization gives the conditions:

$$
\begin{gathered}
\frac{\partial F}{\partial x_{A}}=2 N_{A} Y \gamma_{A}+\lambda y_{A}^{2}=0 \\
\frac{\partial F}{\partial x_{B}}=2 N_{B} Y \gamma_{B}+\mu y_{B}^{2}=0 \\
\frac{\partial F}{\partial y_{A}}=2 \lambda x_{A} y_{A}-\nu N_{A}=0 \\
\frac{\partial F}{\partial y_{B}}=2 \mu x_{B} y_{B}-\eta N_{B}=0 \\
\frac{\partial F}{\partial Y}=2 N_{A} x_{A} \gamma_{A}+2 N_{B} x_{B} \gamma_{B}+4 Y \gamma_{A B}+\nu+\eta=0
\end{gathered}
$$

$\lambda$ and $\mu$ are found from (19) and (20): substituting them into (21) and (22) gives $\nu$ and $\eta$; substituting those into (23) gives the equilibrium condition:

$$
N_{A} x_{A} \gamma_{A}+N_{B} x_{B} \gamma_{B}=2 Y \gamma_{A B}
$$

\section{b) Chemical potentials}

The chemical potential of an $A$ atom near the $A-B$ interface is found by inserting a slab of $A$ with thickness $\Delta x_{A}$ along one of the interfaces of the repeat unit. The number of atoms added is 
$\Delta n_{A, \text { int }}=Y^{2} \Delta x_{A} / \Omega_{A}$, where $\Omega_{A}$ is the atomic volume of $A$. Capillary work is required to extend the grain boundaries of the $A$-surface into the slab:

$$
W_{c, A, i n t}=2 N_{A} Y \Delta x_{A} \gamma_{A}
$$

The chemical potential of $A$ near the $A B$ interface is then:

$$
\mu_{A, i n t}=\frac{W_{c, A, i n t}}{\Delta n_{A, i n t}}=\frac{2 \gamma_{A} \Omega_{A}}{y_{A}}
$$

Similarly, by symmetry,

$$
\mu_{B, i n t}=\frac{2 \gamma_{B} \Omega_{B}}{y_{B}}
$$

These are equivalent to Equation (13) for $\gamma_{y}=\gamma_{z}$.

In order to determine the chemical potentials for atoms near the grain boundaries a slab of thickness $\Delta y$, is placed on an edge of the repeat unit that is parallel to the boundaries, e.g. the top of Figure 4. Since each of the grains is extended with its own atom species, the slab contains $\Delta n_{A, g b}=$ $x_{A} Y \Delta y / \Omega_{A} A$-atoms and $\Delta n_{B, g b}=x_{B} Y \Delta y / \Omega_{B} B$-atoms. The capillary work required is:

$$
W_{c, g b}=N_{A} x_{A} \Delta y \gamma_{A}+N_{B} x_{B} \Delta y \gamma_{B}+2 Y \Delta y \gamma_{A B}
$$

The first and second terms arise from the extensions of the grain boundaries into, respectively, the $A$ and $B$ layers, and the last term from the extension of the two interfaces associated with the repeat unit (one full one in the middle, two outer ones shared). The chemical potentials of $A$ and $B$ in the grain boundary must obey the relation:

$$
W_{c, g b}=\Delta n_{A, g b} \mu_{A, g b}+\Delta n_{B, g b} \mu_{B, g b}
$$

It is obvious how the first two terms in Equation (28) should be assigned to each chemical potential. The partitioning of the last term, however, needs a closer look. In equilibrium the chemical potentials of $A$ and $B$ in the grain boundaries are known, since they must be equal to those in the interface. given by Equations (26) and (27):

$$
\begin{aligned}
& \mu_{A, g b}^{e q}=\mu_{A, i n t}^{e q}=\frac{2 \gamma_{A} \Omega_{A}}{y_{A}} \\
& \mu_{B, g b}^{e q}=\mu_{B, i n t}^{e q}=\frac{2 \gamma_{B} \Omega_{B}}{y_{B}}
\end{aligned}
$$

Therefore:

$$
\Delta n_{A, g b} \mu_{A, g b}^{e q}=2 N_{A} x_{A} \Delta y \gamma_{A}
$$

Comparison with the first term of Equation (28) shows that half of this quantity arises from the extension of the grain boundaries into the $A$-layer, the other half must therefore be the part of the work to extend the interfaces to be assigned to the $A$-atoms. By the same argument, $N_{B} x_{B} \Delta y \gamma_{B}$ must be the part of that work to be assigned to the $B$-atoms. Indeed, the equilibrium condition (24) shows that the two contributions add up to give the total work to extend the interfaces. The ansatz central to of this calculation, now, is that the same partitioning also holds for non-equilibrium dimensions, or that:

$$
\Delta n_{A, g b} \mu_{A, g b}=N_{A} x_{A} \Delta y \gamma_{A}+2 Y \Delta y \gamma_{A B} \frac{N_{A} x_{A} \gamma_{A}}{N_{A} x_{A} \gamma_{A}+N_{B} x_{B} \gamma_{B}}
$$


where the first term is the obvious contribution from the extension of the grain boundaries and the second one is the partitioned interface work. Therefore:

$$
\mu_{A, g b}=\frac{\gamma_{A} \Omega_{A}}{y_{A}}\left[1+\frac{2 Y \gamma_{A B}}{N_{A} x_{A} \gamma_{A}+N_{B} x_{B} \gamma_{B}}\right] .
$$

The same expression, with substituted subscripts, holds for $\mu_{B, g b}$. Note that in equilibrium, where Equation (24) holds, Equation (33) reduces appropriately to Equation (27). Comparison of Equation (33) and its equivalent for $B$ with Equation (26) and (27) shows that the partitioning, as defined here, can also be expressed as:

$$
\frac{\mu_{A, i n t}}{\mu_{B, i n t}}=\frac{\mu_{A, g b}}{\mu_{B, g b}}
$$

A special case that often applies [6-8] is that of epitaxial growth with the columnar grains extending through the layers, or: $y=y_{A}=y_{B}$ and $N_{A}=N_{B}=1$. Equation (33) then becomes:

$$
\mu_{A, g b}=\frac{\gamma_{A} \Omega_{A}}{y}\left[1+\frac{2 y \gamma_{A B}}{x_{A} \gamma_{A}+x_{B} \gamma_{B}}\right]
$$

As a check on the magnitude of these potentials, consider, for example such a multilayer with a grain size $y$ that is smaller than the value required by the equilibrium condition (24). In that case, for both species, $\mu_{g b}<\mu_{i n t}$. As a result, matter flows from the interfaces to the grain boundaries, making the grains larger and thinner, as required for equilibrium.

\section{FINAL REMARKS}

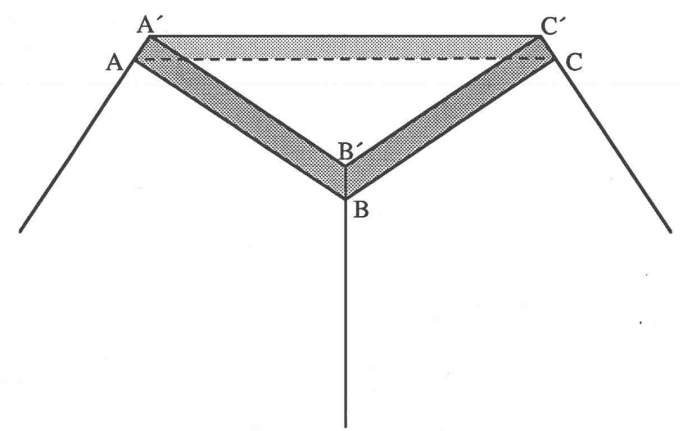

Figure 5: lllustration of the method for determining the chemical potential of the atoms on a facet with nonorthogonal neighbors. The capillary work has positive and negative contributions (see text).

The chemical potentials derived in the previous sections can be used, together with the mechanical contribution, to analyze diffusional flow. The fluxes governing this process are driven by the differences in these chemical potentials. As a result, under certain conditions, the problem of partitioning addressed in Section 4 may not arise - in that any partitioning may lead to the same overall strain rate $[7,8]$.

Shapes with symmetries other than the orthorhombic and tetragonal ones described here can be treated in a very similar way. Herring [11] has pointed out that the layer added to determine the 
chemical potential on a face should be bounded by the extension of the neighboring faces of the grain, as illustrated by Figure 5. The contributions in that specific case correspond to a negative one corresponding to the decrease in area of the facet under consideration ( $\left.\mathrm{ABC} \rightarrow \mathrm{A}^{\prime} \mathrm{B}^{\prime} \mathrm{C}\right)$, and three positive ones, corresponding to the slanted rims of the neighboring facets (AA'BB', $\mathrm{AA}^{\prime} \mathrm{CC}$, $\mathrm{BB}$ 'CC).

\section{ACKNOWLEDGEMENTS}

I thank Dan Josell for many useful discussions. This work has been supported by the Office of Naval Research under contract number N00014-J-91-1281

\section{REFERENCES}

1. Udin, H., Shaler, AJ., and Wulff, J.: AIME: Met. Trans. 185 (1949), 186.

2. Udin, H.: Trans. AIME 189 (1951), 63.

3. Jones, H. and Leak, G.M.: Acta Metall. 14 (1966), 21.

4. Hondros, E.D.: Proc. Roy. Soc. A286 (1965),479.

5. Fisher, J.C. and Dunn, E.G.: in Imperfections in Nearly Perfect Crystals, Wiley, NY, (1952), 317.

6. Josell, D. and Spaepen. F.: Mat. Res. Soc. Symp. Proc. 239 (1992), 515.

7. Josell, D.: Ph.D. Thesis, Harvard University (1992).

8. Josell, D. and Spaepen, F.: Acta Metall. 41 (1993), 3007 \& 30017.

9. Gibbs, J.W.: The Collected Works of J. Willard Gibbs, Yale University Press, New Haven (1948), 148-219.

10. Herring, C.: J. Appl. Phys. 21 (1949), 437.

11. Herring, C.: in The Physics of Powder Metallurgy, ed. by W.E. Kingston, McGrawHill, NY (1951), 143.

12. Larche, F. and Cahn, J.W.: Acta Metall. 33 (1985), 331.

13. Mullins, W.W.: in Metal Surfaces, ASM, Metals Park, OH (1962), 17.

14. Herring, C.: Phys. Rev. 82 (1951), 87. 